\title{
On the treatment of neutron scattering in the resonance range
}

\author{
R. Dagan ${ }^{1}$, J. Rowlands ${ }^{1}$, A. Courcelle ${ }^{2}$, and C.R. Lubitz ${ }^{3}$ \\ 1 Institute for Reactor Safety, Forschungszentrum Karlsruhe, Postfach 3640, 76021 Karlsruhe, Germany \\ 2 CEA/Cadarache, DEN/DEC/SESC/LSC, 13108 Saint-Paul-lez-Durance, France \\ 3 Knolls Atomic Power Laboratory, Schenectady, NY, USA
}

\begin{abstract}
An improved double-differential resonance-dependent scattering kernel is presented and its impact on criticality, Doppler Effect, absorption, and inventory of heavy isotopes for a typical unit cell is confirmed again to be significant. The influence of solid state effects is investigated. The way the resonant crystal lattice model based on a 4-point correlation function differs from the free gas scattering kernel is discussed and the differences are shown to be small.
\end{abstract}

\section{Introduction}

In deterministic calculations of the neutron slowing down spectrum in the resonance range a particularly simple approximation is usually made for the secondary energy distribution of scattered neutrons (the transfer kernel). This treats the scattering as uniform over the range $\alpha E \rightarrow E$ where $\alpha=$ $[(A-1) /(A+1)]^{2}$ and $\mathrm{A}$ is the mass-ratio between the target and the neutron. It ignores the effects of the thermal motion and the associated up-scattering effects. In some Monte Carlo calculations the above 0 degree $\mathrm{K}$, asymptotic kernel is extended for certain resonances, as a function of the temperature, and includes the effects of thermal motion on the scattering distributions in the resonance range. Yet this approach uses a form of secondary distribution which applies only to a scattering cross-section which is constant in energy.

In this study we discuss an improvement of this approximate scattering kernel to include the effect of resonances and temperatures on the scattering kernel, and also discuss the impact of the solid state effects on the kernel. This is done by analysing the quantum crystal lattice formalism for resonant scattering by a bound nucleus developed by Word and Trammell. The results of former studies are discussed and new solution methods in the limit of the short time approximation are presented and compared with the free gas energy dependent scattering kernel.

The applicability of the new resonant scattering kernel is also discussed relative to experiment, which might assess how well it predicts the secondary distribution. Since transmission and capture experiments do not focus on this important aspect, a new dedicated experiments is suggested to evaluate directly the predicted angular distribution of the model.

\section{Free gas treatment of resonant scattering}

Wigner and Wilkins [3] used a two-body kinematics approach for potential scattering and developed an isotropic temperature dependent scattering kernel. Brown and St. John [4] extended the model to an energy dependent cross section using an exponential function for the scattering cross section of $\mathrm{D}_{2} \mathrm{O}$. Blackshaw and Murray [5] looked further at strongly energy dependent cross sections. Their study was followed by Ouisloumen and Sanchez [6] who developed expressions for the Legendre coefficients of the scattering kernel and calculated the isotropic $\left(\mathrm{P}_{0}\right)$ resonance dependent scattering kernel.

This study showed that the improved kernel, which allows for the energy dependence of the cross-sections, shifts more neutrons into the energy range of the resonance. The resonance absorption is thus increased, with the effect being relatively higher at higher temperatures. The above isotropic kernel was introduced into fine flux calculation codes for the resolved resonance region. Qualitative results given by Bouland, Kolesov and Rowlands [2] and confirmed independently by Dagan and Rothenstein [11] showed an increase of the absorption in the resonances by about $1 \%$ at $1200 \mathrm{~K}$. The Doppler Effect in this temperature range was also increased by about $12 \%$. The double differential scattering kernel developed by Rothenstein and Dagan [1] allowed for the inclusion of the angular distribution, which is important for heterogeneous designs. Further mathematical improvements by Rothenstein [12] enabled the introduction of a new kernel (eq. (1)) into the NJOY [13] processing code:

$$
\begin{aligned}
& \sigma_{s}^{T}\left(E \rightarrow E^{\prime}, \vec{\Omega} \rightarrow \overrightarrow{\Omega^{\prime}}\right)=\frac{1}{4 \pi E} \sqrt{\frac{A+1}{A \pi}} \int_{\varepsilon_{\max }}^{\infty} d \xi \int_{\tau_{0}(\xi)}^{\tau_{1}(\xi)} d \tau\left(\frac{\xi+\tau}{2}\right) \\
& \times\left(\sigma_{s}^{t a b}\left[\left(\frac{A+1}{A^{2}}\right) \frac{(\xi+\tau)^{2}}{4} k_{B} T, 0\right]\right) \\
& \times \exp \left(v^{2}-\left[\frac{(\xi+\tau)^{2}}{4 A}+\frac{(\xi-\tau)^{2}}{4}\right]\right) \times\left(\frac{\varepsilon_{\max } \varepsilon_{\min }(\xi-\tau)^{2}}{B_{0} \sin \hat{\varphi}}\right) .
\end{aligned}
$$

The integration variables $(\xi, \tau)$ are introduced by rotating the velocity plane of integration of the original variables $(\mathrm{t}, \mathrm{x})$, where: $t=u \sqrt{(A+1)} ; x=c \sqrt{(A+1)}$. " $u$ " and " $c$ " are the velocity of the neutron in the centre of mass (C.O.M.) system and the velocity of the C.O.M. respectively and sigmatab (tabulated) is the energy dependent cross section being broadened. $\varepsilon_{\max }^{2}=(A+1) \max / \min \left(E, E^{\prime}\right)$. (For the rest parameters see [12].) 
This new kernel has several important advantages. It is mathematically consistent and in accordance with the BROADR module (Doppler broadening) of NJOY.

Further, due to the reduction of the former quadric expressions to quadratic equations the time needed for the numerical solution was reduced considerably. The accelerated solver allowed for the generation of probability $(S(\alpha, \beta))$ tables in the same manner as it is done for the light bound nucleus scattering kernel, which is introduced as an input for MCNP [14]. About 1000 probability tables were prepared at each selected temperature covering the first $8 \mathrm{~S}$ resonances of U238 appropriately. The introduced tables enabled the evaluation of the resonance scattering kernel impact for any core configuration. For a typical light water reactor unit cell at a fuel temperature of $1200 \mathrm{~K}$ it was seen that through the enhanced resonant absorption the criticality value decreased by about $440 \mathrm{pcm}$ compared to the reference simulation with the current scattering treatment in MCNP, which uses the constant cross-section kernel either in its asymptotic form or in its temperature dependent form. The Doppler Effect was increased by about $13 \%$ between $800 \mathrm{~K}$ and $1200 \mathrm{~K}$. The additional absorption in U238 leads to the breeding of more Pu239. Therefore the inventory of the PU239 was seen to grow by $2 \%$ after $50 \mathrm{MWD} / \mathrm{Kg}$. In figure 1 the same results are introduced for a very heterogeneous system like TRISO particle in a HTGR pebble bed. In addition to the energy upscattering effects, governing the changes mentioned above, the deviations of the angular distribution of the new $S(\alpha, \beta)$ tables in comparison with the MCNP reference resonant treatment are expected to be well pronounced.

The initial reduction in criticality shown in figure 1 is about $1.5 \%$ at $1800 \mathrm{~K}$ (at $1200 \mathrm{~K}$ it is about $1.1 \%$ ). During the burn up cycle the differences between the MCNP reference solution and the improved case with the new tables becomes smaller. The reason can be attributed to the relative increase in PU239 inventory and to a minor extent the increase of the U235 inventory. As can be seen in figure 1 the changes in the PU239 inventory reaches almost $5 \%$ at $1800 \mathrm{~K}$ by comparing the two different treatments of the scattering kernel.

\section{Possible measurements of scattering distributions}

The results of the former section emphasize the need for dedicated experiments which could measure the up-scattering as well as the angular distribution.

In order to assess the validity of the new kernel at ambient temperature, with regard to the importance of solid state effects (discussed in the next section), a Time of Flight (TOF) measurement was suggested by Y. Danon [15].

In figure 2 it is seen that neutrons counted in the detector are those which collided with the target and were scattered at a specific angle and energy, contrary to transmission measurements. In this way the angular distribution prediction of a certain model can be directly assessed. A TOF simulation at $300 \mathrm{~K}$ was performed using the MCNP code. The integrated flux reaching a point detector 25 meters from the U238 sample is higher by about $4.3 \%$ using the new $S(\alpha, \beta)$ tables instead of the reference asymptotic kernel in MCNP. Real experiments

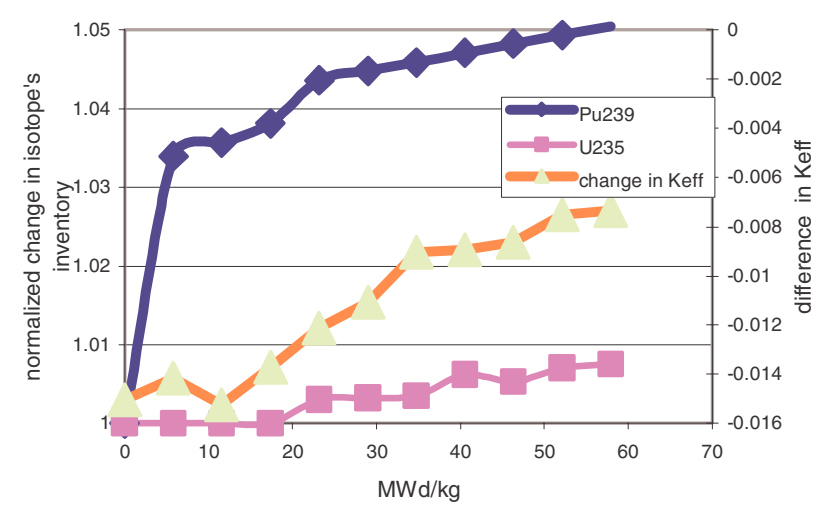

Fig. 1. Differences in Keff and in the Pu239 and U235 inventory due to the introduction of $\mathrm{S}(\mathrm{a}, \mathrm{b})$ tables at $1800 \mathrm{~K}$ instead of the reference method of MCNP for HTGR TRISO particles in a pebble bed unit cell.

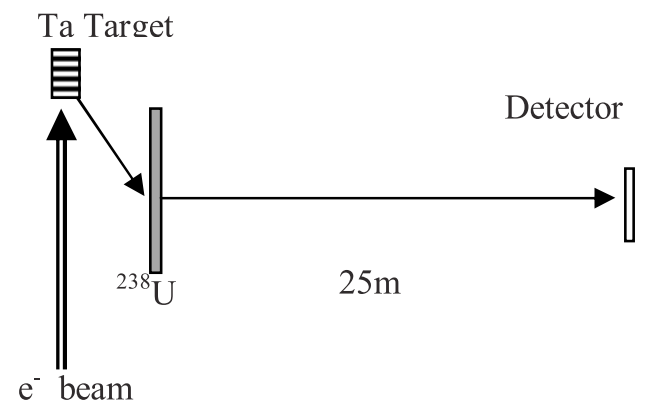

Fig. 2. A sketch of the proposed experimental geometry. The Ta Target (neutron source) and U238 sample distance is $15 \mathrm{~cm}$ and the nominal scattering angle is $45 \mathrm{deg}$. An angle of $135 \mathrm{deg}$ can be obtained by moving the sample to left side of the Ta target [15].

might therefore corroborate the applicability of the free gas model and assess the importance of solid state effects in resonant scattering.

Another advantage which arises from figure 2 is the possibility of evaluating the thickness effect on the angular distribution. In [21] it was shown that changing the thickness of the sample from $4 \times 4 \times 0.125 \mathrm{~cm}$ to $10 \times 10 \times 0.25 \mathrm{~cm}$ leads to differences of about $50 \%$ at $1200 \mathrm{~K}$ in the neutron density reaching the detector, emphasizing the importance of the correct angular distribution treatment and the need of such dedicated experiments. The technical feasibility of performing such an experiment, which will be comparable to the above MCNP simulations, was confirmed by [15].

\section{Solid state effect in resonant scattering}

In his 1939 paper [24] on the capture of neutrons by atoms of a crystal, Lamb developed an expression for resonance capture which applies when the nuclei are bound in a crystal. He showed that in the approximation of the weak binding limit the absorption curve has the same form for an atom bound in a crystal as in a free gas, but calculated assuming a temperature which corresponds to the average energy of the nuclei per vibrational degree of freedom. For room temperature, and 
higher, this effective temperature is just a few degrees higher than the thermodynamic temperature and can be represented by the approximate form:

$$
\mathrm{T}_{\mathrm{eff}} \approx \mathrm{T} \cdot\left[1+(1 / 20) \cdot\left(\Theta_{\mathrm{D}} / \mathrm{T}\right)^{2}\right]
$$

where $\Theta_{D}$ denotes the "Debye temperature", which has been estimated to be about $250 \mathrm{~K}$ for uranium in both uranium metal and uranium oxide form.

In recent years there have been several studies which have shown that for resonances of heavy nuclei, such as uranium, neptunium and plutonium, at energies below about $10 \mathrm{eV}$, it is necessary to take into account the crystal binding effects to get an accurate fit to the shape of the resonance as measured in transmission measurements $[16,17]$. The resonance parameters obtained in such a fit can differ by several percent from those obtained using a free gas model.

However, although the parameters obtained by fitting the measurements can differ between the gas model and the solid state model the difference between reactor calculations of U238 capture rates (made using the two types of model to generate the cross-section curve used in the calculations) are negligibly small $\sim 0.1 \%$ [18]. For resonances at energies above about $10 \mathrm{eV}$ and at temperatures above ambient it is expected that the Lamb approximation will also be valid for the calculation of scattering effects and it is at these energies and at higher temperatures that the effects are important.

Regarding the double differential scattering cross section or scattering kernel, the classic paper of Van-Hove [7] provided a general quantum formalism to study solid state effects for potential scattering. The potential scattering kernel is proportional to $S(\alpha, \beta)$ : the Fourier transform of a 2-point correlation function.

For resonant scattering, the Van-Hove formalism was extended by Word and Trammell [8]. The resonant kernel involves a three-fold integral of a 4-point correlation function involving the time parameters $\mathrm{t}, \mathrm{t}^{\prime}$ and $\mathrm{T}$. The time parameter $\mathrm{T}$ is related to the potential scattering (Van-Hove [7]), whereas $\mathrm{t}$ and $\mathrm{t}$ ' the connected to the resonance character of the reaction. Shamaoun et al. [19] showed that by applying the short collision time approximation (valid for higher energies and temperatures), the crystal-lattice kernel reduces to the free gas expression with the same effective temperature as derived by Lamb for the case of resonance capture.

In 2001, in his independent study of resonance scattering, Naberejnev [20], again starting from the expressions developed by Trammell et al. developed a similar expression to that presented by Shamaoun et al. His work is the only one to have attempted to provide a numerical solution for the case of scattering by nuclei bound in solids. In his study Naberejnev proposed what he called the Uncoupled Phonon Approximation, (UPA). Within this approximation, the double differential cross section is found to be a product of the classic Van-Hove constant cross-section scattering function $S(\alpha, \beta)$ and a second term (the UPA cross section) which depends on the crystal dynamics but has only a small effect on the scattering energy distribution. The result is significantly different from the free gas resonant scattering distribution.

The approximation made by Naberejnev, which is to neglect the terms in $t$ and $t^{\prime}$ when combined with $T$ were introduced into equation (62) of Shamaoun et al.
In the short collision time approximation Shamaoun and Summerfield expand the sine and cosine terms $[\sin ($ at $) \sim$ at; $\left.\left.\{1-\cos (a t)\} \sim(a t)^{2} / 2\right\}\right]$, leading to the following expression:

$$
\begin{aligned}
& \exp \left[\gamma\left(T, t^{\prime}, t\right)\right]=\exp \left[-\frac{\Delta K^{2}}{2 M_{L}}\left(i T \hbar+\frac{T^{2}}{\beta_{e f f}}\right)\right] \\
& \times \exp \left[-i\left(t^{\prime}-t\right) \frac{\hbar K_{i}^{2}}{2 M_{L}}-\frac{\left[K_{f}\left(t-t^{\prime}\right)\right]^{2}}{2 M_{L} \beta_{e f f}}\right]
\end{aligned}
$$

where $\mathbf{K}_{\mathrm{i}}$ and $\mathbf{K}_{\mathrm{f}}$ are the momentum vectors of the incident and scattered neutrons and $\Delta K$ is the difference between them. The squared values $\mathrm{K}^{2} / 2 \mathrm{M}$, give the corresponding energies. $\beta_{\text {eff }}^{-1}$ is the effective temperature in energy units determined by the crystal phonon spectrum [19].

It is seen that the UPA formalism, as applied to U-238 resonances at ambient temperature, does not go to the expected free gas model resonance scattering (as explained in section 2) in the short time approximation, but rather to the constant cross section scattering secondary distribution (first term on the right hand side of equation (2)) multiplied by factors which depends on the Doppler broadening of the resonances (second term on the right hand side of equation (2)). The results obtained by the UPA formalism were compared (for the same energy as in [20]) to calculation based on the potential scattering free gas kernel using the solution technique mentioned in section 2 . The differences were very small in view of the different solution methods [23].

A different decoupling scheme was developed in a recent work by A. Courcelle et al. [21,22] to improve on the previous approximation. The kernel is still split into the $S(\alpha, \beta)$ and a UPA cross section but the latter term was calculated to provide the correct limit at short collision time. As shown in figure 3, the resulting scattering kernel in U238 at room temperature is very close to the Free gas Model developed by Ouisloumen et al. and Rothenstein and Dagan. The only significant difference is the presence of the individual phonon transition peaks near to the incident neutron energy. Therefore, contrary to the UPA approximation, the new approach predicts very small solid state effect for U238 at room temperature.

\section{Conclusion}

We have shown that the new treatment for the resonant dependent ideal gas scattering kernel causes noticeable changes in criticality, absorption, Doppler Effect and fuel inventory, in particular for very heterogeneous core designs. Moreover the importance of the angular distribution was emphasized by the TOF simulation at $300 \mathrm{~K}$. New types of experiments, like those suggested by [15], are highly important because they deal directly with the angular distribution of the scattered neutrons. Consequently the differentiation between the solid state effects and the free gas model could be illuminated in the vicinity of the resonances not only based on the different capture as it is usually done in the current measurements but rather on the different flight direction of the neutrons. Together with the existing experimental results, such measurements could provide a validation of the scattering kernel. 


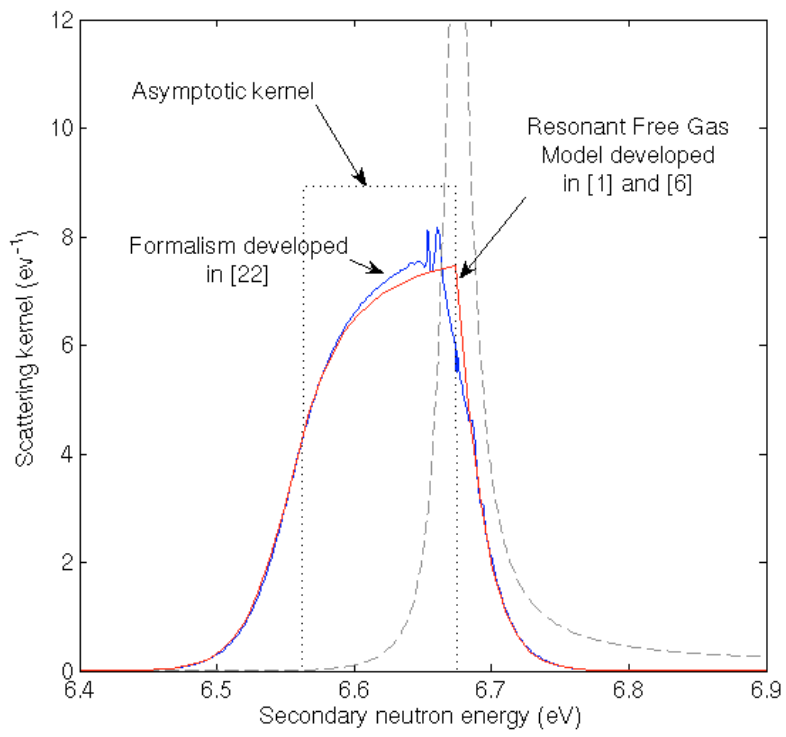

Fig. 3. Comparison of scattering kernel calculated with a free gas model formalism and an approximate solid state model developed in [22] at $\mathrm{T}=300 \mathrm{~K}$ for an incident neutron energy of $6.674 \mathrm{eV}$. The elastic peak is not displayed. The dashed curve presents qualitatively the shape of the absorption cross section.

The analysis performed for the solid state effects points out the possible correlation between the free gas model and the resonant dependent crystal lattice model. The 4 point correlation scattering function given by [8] as solved by [22] was compared to the new treatment of the resonant free gas scattering kernel and it was shown that solid state effects are relatively small when treated using this (albeit approximate) method. In addition we showed that the different approximation suggested by [20] leads essentially to the potential scattering kernel. Therefore, it is considered that the improved free gas kernel is a good first approximation for calculating the resonant scattering kernel. The impact of additional predicted absorption due to the up scattered neutrons into the resonances should be analysed in view of the current measurements from which the specific resonance parameters are specified. Such an analysis should account also for the shifted angular distribution which was shown to have a significant impact in the simulation of the scattering measurements.
However it is clear that more investigation is needed to:

(1) Solve the strongly oscillatory 4 point correlation function of [8] in its incoherent approximation form,

(2) Relax the constraint of the short collision time approximation in order to improve the resonance treatment and in particular the Doppler broadening,

(3) Clarify how accurate the free gas model, which is widely used in the analysis of experimental results, really is.

\section{References}

1. W. Rothenstein, R. Dagan, Ann. Nucl. Energy 25, 209 (1998).

2. O. Bouland, V. Kolesov, J. Rowlands, CEA Report SPRC/ $\mathrm{LEPh} / 93 / 219$ (1993).

3. E.P. Wigner, E.J. Wilkins, AECD-2275, Clinton Laboratory (1944).

4. H.D. Brown, D.S. St. John, Oak-Ridge report DP-33 (1954).

5. G.L. Blackshaw, R.L. Murray, Nucl. Sci. Eng. 27, 520 (1967).

6. M. Ouisloumen, R. Sanchez, Nucl. Sci. Eng. 107, 189 (1991).

7. L. Van Hove, Phys. Rev. 95, 249 (1954).

8. R.E. Word, G.T. Trammell, Phys. Rev. B 24, 2430 (1981).

9. D.G. Nabarejnev, Ann. Nucl. Energy 28, 1 (2001).

10. A.I. Shamaoun, G.C. Summerfield, Ann Nucl. Energy 17, 229 (1990).

11. R. Dagan, W. Rothenstein, Proc. of ICENES Meeting (1998).

12. W. Rothenstein, Ann. Nucl. Energy 31, (2004).

13. R.E. Macfarlane, D.W. Muir, 1994. The NJOY Nuclear Data Processing System Version 91, LA-12740-M.

14. J.F. Briesmeister, Editor 1997. MCNP-A General Monte Carlo N-Particle Transport Code, LA-12625-M.

15. Y. Danon, RPI (2006) (private communication).

16. Seidel, Seeliger, Meister, Mittag, and Pilz INDC(GDR)50/L(1989).

17. V. Gressier, D.G. Naberejnev, C. Mounier, Ann. Nucl. Energy 27 (2000).

18. D.G. Naberejnev, C. Mounier, R. Sanchez, JEF/DOC-750.

19. A. Shamaoun, G. Summerfield, Ann. Nucl. Energy 17, 229 1990.

20. D. Nabarejnev, Ann. Nucl. Energy 28, (2001).

21. R. Dagan. A. Courcelle, J. Rowlands, JEFF-DOC 1162 (2006).

22. A. Courcelle et al. (unpublished).

23. R. Dagan, Ann. Nucl. Energy 32, 367 (2005).

24. W.E. Lamb, Phys. Rev. 55, 750 (1939). 